\title{
The AGN-Starburst Connection traced by the Nitrogen Abundance
}

\author{
Kenta Matsuoka ${ }^{1,2}$, Tohru Nagao, Alessandro Marconi, \\ Roberto Maiolino, and Yoshiaki Taniguchi \\ ${ }^{1}$ Graduate School of Science and Engineering, Ehime University, 2-5 Bunkyo-cho, Matsuyama \\ 790-8577, Japan \\ ${ }^{2}$ Department of Astronomy, Kyoto University, Kitashirakawa-Oiwake-cho, Sakyo-ku, Kyoto \\ 606-8502, Japan - email: matsuoka@kusastro.kyoto-u.ac.jp
}

\begin{abstract}
The connection between the active galactic nuclei (AGNs) and star formation activity is one of the most important issues in understanding the coevolution of supermassive black holes (SMBHs) and galaxies. In our recent study, by using SDSS quasar spectra we found that the emission-line flux rations involving a nitrogen line correlate with the Eddington ratio. This correlation suggests that the mass accretion in quasars is associated with a post-starburst phase, when AGB stars enrich the interstellar medium with the nitrogen. Moreover, we focused on nitrogen-loud quasars, that have prominent emission lines of the nitrogen, to investigate whether this argument is correct. In this symposium, we present our results regarding the relation between the star formation and feeding to SMBHs traced by the nitrogen abundance.
\end{abstract}

Keywords. galaxies: active, galaxies: evolution, galaxies: nuclei, quasars: emission lines

\section{Introduction}

The mass of supermassive black holes (SMBHs) at center of galaxies is correlated tightly with the mass of their bulges (e.g., Magorrian et al. 1998). This correlation suggests that the growth of SMBHs is connected with that of their host galaxies, that is called "the coevolution of SMBHs and galaxies". In the previous studies, it has been reported that the activity of active galactic nucleus (AGN), i.e., the mass accretion to the SMBH, is related to the star formation activity. Recent studies on local AGNs have shown that nuclear star formation and AGN activity are not coeval (e.g., Davies et al. 2007). More specifically, the black hole accretion appears to occur in post-starburst nuclei, with a delay of $\sim 10^{8}$ years from the starburst. Probably during the active starburst phase powerful supernova explosions expel the circumnuclear gas preventing it to reach the black hole, while the more gentle winds of AGB stars occurring on time scales of $10^{8}$ years are capable of stirring the interstellar matter, making it loose angular momentum and then feed the AGN. In order to investigate the relation between the star formation and ignition timing of AGNs, and to assess which stellar population in a host galaxy regulates the growth of SMBHs, it is incredibly important to pursue whether this argument is correct, leading to the understanding of the coevolution.

\section{The relation between the Eddington ratio and nitrogen abundance}

By using optical spectra of 2383 quasars at $2.3<z<3.0$, we investigated the dependence of the emission-line flux ratios as metallicity on the Eddington ratio (Matsuoka et al. 2011); we measured the emission-line flux ratios of Nv/CIV, Nv/HeII, (Sirv+OIV])/ CIV, and AlıII/CIV, that are sensitive to metallicity of the broad-line region (BLR). As the result, we found that the Eddington ratio depends on the emission-line flux ratios involving $\mathrm{Nv}$, while it does not correlate with the other emission-line flux ratios, i.e., 


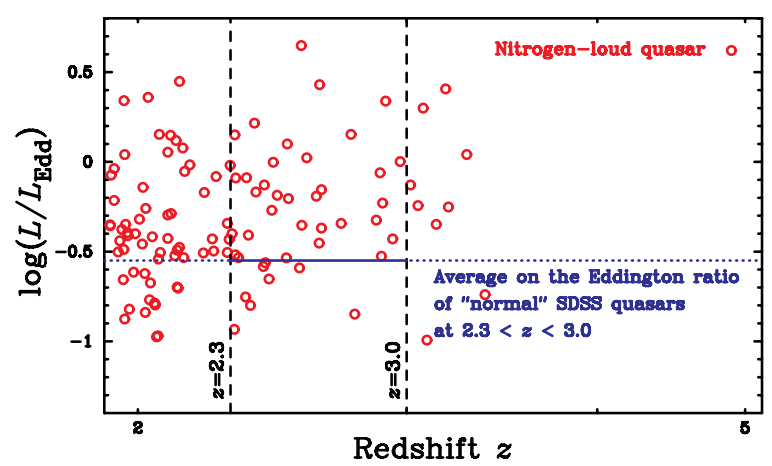

Figure 1. The redshift distribution in the Eddington ratio of N-loud quasars. The horizontal line shows the average on the Eddington ratio of SDSS quasars at $2.3<z<3.0$ as "normal" quasars.

(Sirv+OIv])/CIV and AlıII/CIV (see Fig. 6 in Matsuoka et al. 2011). We suggest that the correlation between the Eddington ratio and the line ratios including the nitrogen line, is tracing a delay of the mass accretion to $\mathrm{SMBHs}$ relative to the onset of nuclear star formation of about $10^{8}$ years reported by Davies et al. (2007), which is the time scale required for the nitrogen enrichment. This result supports the connection between the AGN activity and post starburst, and means that AGNs in higher accretion phase, i.e., SMBHs in more rapid growth, have more nitrogen-rich gas clouds.

\section{Nitrogen-loud quasars}

Osmer \& Smith (1980) discovered unusual quasar at $z=1.96$ that has prominent nitrogen emission lines; this is called nitrogen-loud quasar (hereafter, N-loud quasar). Baldwin et al. (2003) claimed that the unusual nitrogen emission in the quasar is likely due to high metallicity in the BLR of the quasar; the metallicity measured from line strength ratios involving nitrogen lines is $\sim 15$ times the solar abundance. However, such extremely high metallicity is very hard to be reconciled with galaxy chemical evolutionary models (e.g., Hamann \& Ferland 1993). Then, we suspect that N-loud quasar is in especially high accretion phase with the nitrogen-rich gas clouds, not metal-rich gas clouds. Recently, in order to examine whether the N-loud quasars have really extremely high metallicity, we have investigated the narrow-line region (NLR) metallicity of N-loud quasars by using [OIII] $\lambda 5007$ emission line; if the NLR metallicity is low, [OIII] line should be very week due to the low equilibrium temperature of the ionized gas owing to significant metal cooling. Then, we found that the [OIII] emission line of N-loud quasars is quite strong, suggesting that the NLR in N-loud quasars is not extremely metal rich at all (e.g., Araki et al. 2012). Moreover, by investigating the Eddington ratio of N-loud quasars, we found that their Eddington ratio is significantly higher than the average of "normal" SDSS quasars as shown in Figure 1 (Matsuoka et al. in prep.). These results are consistent with Matsuoka et al. (2011), meaning that N-loud quasars are in the post-starburst phase.

\section{References}

Araki, N., Nagao, T., Matsuoka, K. et al. 2012, A\& A, 543, A143

Baldwin, J. A., Hamann, F., Korista, K. T. et al. 2003, ApJ, 583, 649

Davies, R. I., Sánchez, F. M., Genzel, R. et al. 2007, ApJ, 671, 1388

Hamann, F. \& Ferland, G. 1993, ApJ, 418, 11

Magorrian, J., Tremaine, S., Richstone, D. et al. 1998, AJ, 115, 2285

Matsuoka, K., Nagao, T., Marconi, A. et al. 2011, A\&A, 527, A100

Osmer, P. S. \& Smith, M. G. 1980, ApJS, 42, 333 